\title{
Performance of a low cost laser scanner using a granite square gauge
}

\section{Performance d'un scanner laser à faible coût à l'aide d'une jauge carrée de granite}

\author{
Antonio Piratelli-Filho, Nabil Anwer' ${ }^{2}$, Charyar Mehdi Souzani², Rosenda Valdes Arencibia ${ }^{3}$ and Lucas Peres dos Reis ${ }^{1}$ \\ ${ }^{1}$ Universidade de Brasília, UnB, Faculdade de Tecnologia, Depto. Engenharia Mecânica, Brasília, DF, Brazil \\ ${ }^{2}$ LURPA, École Normale Superieure - ENS Cachan, 61, av. du Président Wilson, Cachan, France \\ ${ }^{3}$ Universidade Federal de Uberlândia - UFU, Faculdade de Engenharia Mecânica, Uberlândia, MG, Brazil
}

\begin{abstract}
This work deals with performance verification of a low cost laser scanner with a granite square gauge. This gauge has well finished surfaces and dimensional precision, enough for verification of a low cost laser scanner. The square was initially measured with the scanner and a cloud of points was saved in a file. A filtering method was applied to extract noise from data and the flatness deviation of each plane, together with the angle between planes, were determined. MatLab software was used as a tool to determine the deviations and angles. Analysis of normality of data was carried out with normal probability plot graphs. Results were compared and were below from scanner manufacturer ones. It was observed that deviations were above stated in manufacturer manual.
\end{abstract}

Key Words: laser scanner, performance, square gauge

Mots Clés: laser scanner, performance, étalon carré

\section{Introduction}

Performance evaluation of laser scanner 3D has been under investigation in search of knowledge about its errors and uncertainty. Current techniques involves the use of different types of gauges like spheres and gauge blocks among others [1].

According to Lartigue et al. [2], the parameters density, completeness, noise and accuracy define the quality of a point cloud obtained by digitization instruments. The authors argued that noise and accuracy are linked to quality, as density and completeness are influenced by measurement strategy and object.

Barbero and Ureta [1] compared five different digitization techniques in respect its accuracy. There were used a calibrated sphere, cylinder and gauge block as gauges to perform the measurements.

Zúniga and Piratelli-Filho [3] presented a method to check performance of laser scanner $3 \mathrm{D}$, based on the measurement of a multi-object gauge. This gauge was built having cylinder, cone, rectangle, stairs and circle to verify errors in length and angle measurement.

Alternatives to complex objects are simple ones. Besides, data points considered as outliers, coming from some particular problem during scanning as laser light reflection, must be extracted before determining the performance parameter. Besic et al [4] presented an application to improve the accuracy of a laser line scanner using the median filter. Gestel et al. [5] used a plane to check the performance of laser line scanners, arguing that it is a fast, easy and simple method.

This work deals with performance verification of a low cost three dimensional laser scanner with a granite square gauge. This gauge has well finished surfaces and dimensional precision, enough for verification of a low cost laser scanner. Data filtering was carried out by using statistical boxplot graphics and the performance was determined by the range of deviations in respect to the fitted planes and angle of the square.

\section{Experimental}

The experiments were performed with a 3D laser scanner from NextEngine, model 2020i. This scanner has four laser sources with wavelenght of $0.650 \mathrm{~mm}$. The digitization speed is 50,000 points per second and there are two scanning modes, Macro and Wide, having resolution and accuracy of $200 \mathrm{dpi}$ and $0.127 \mathrm{~mm}$ for Macro and $75 \mathrm{dpi}$ and $0.381 \mathrm{~mm}$ for Wide, respectively. The sofware ScanStudio HD controls the digitization.

The gauge adopted was a calibrated granite square with flatness deviation of $0.002 \mathrm{~mm}$ in planes A and B, with a $95 \%$ expanded uncertainty of $0.001 \mathrm{~mm}$. The angle between planes $\mathrm{A}$ and $\mathrm{B}$ was was reported as $90,0011^{\circ}$ by the calibration certificate. 
Figure 1 shows the scanner and gauge during measurement. It can be seen that 4 laser lines were projected over granite square surfaces that were reflected to the laser camera. Cartesian coordinates of points on surfaces are determined and saved as cloud of points in a txt format file.

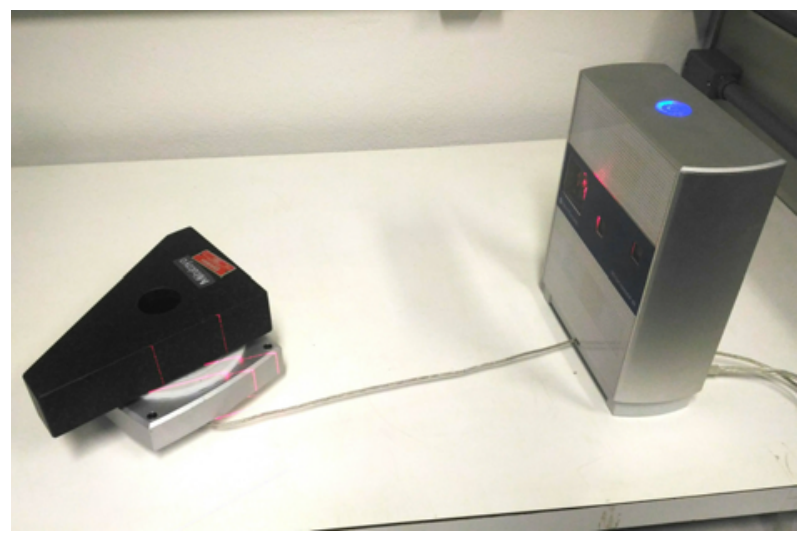

Figure 1. Laser scanner and granite square gauge.

The gauge was measured using the following settings at 3D laser scanner: Positioning at Single; resolution of 200 points/in²; Target at Neutral; Range at Wide. The gauge positioning was selected to acquire all information of both planes and angle in a single measurement, avoiding alignment errors from joining different views.

MatLab software was used to make calculations and graphs. A plane was fitted to each data cloud and the deviations between points and planes were determined, as the angle between planes. Data was checked against normality of deviation distribution on surfaces A and B of the granite square. A statistical filtering method was applied by using the boxplot graphs, in an attempt to reduce the variability associated to scanning operation.

\section{Results and discussion}

A first view of the data point deviations in respect to the planes $\mathrm{A}$ and $\mathrm{B}$ is presented in Figure 2. As observed, the global mean was $0.000 \mathrm{~mm}$, with a standard deviation of $0.104 \mathrm{~mm}$. This result is valid as a preliminary analysis, but considering that range was $1.282 \mathrm{~mm}$, compared to flatness of $0.002 \mathrm{~mm}$ of square and $0.127 \mathrm{~mm}$ from scanner accuracy (manufacturer), it can be considered that noise is a considerable part of results. If flatness and other geometrical features are under evaluation, a filtering method must be applied and new analysis was demanded.

Data from scanner was then exported to complementary analysis using MatLab. Descriptive statistics were mean, stardard deviation and range as $0.000 \mathrm{~mm}, 0.090$ $\mathrm{mm}$ and $1.058 \mathrm{~mm}$ for plane $\mathrm{A}$ and $0.000 \mathrm{~mm}, 0.157$ $\mathrm{mm}$ and $1.360 \mathrm{~mm}$ for plane B. Figures 3 and 4 presents the histograms for planes A and B. As showed, the distribution appears like a normal one, but with some addtional negative deviations in both planes. These values increase the range and standard deviation and can be associated to noise in scanned data.

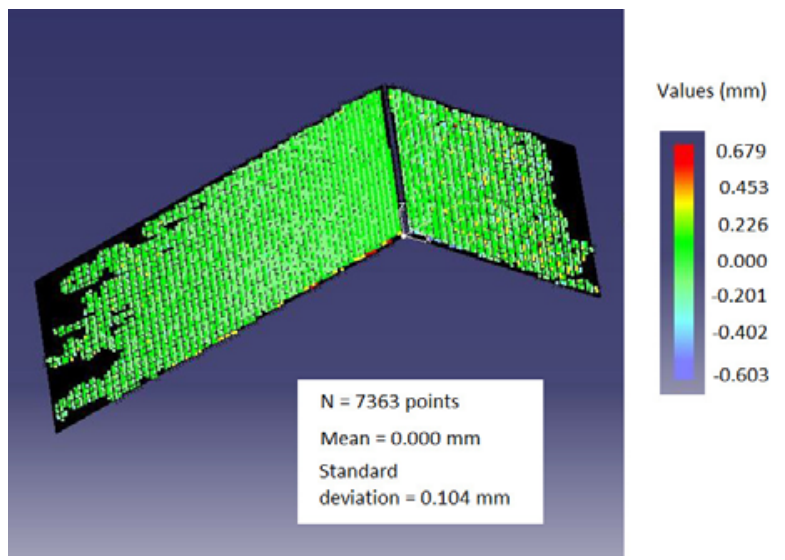

Figure 2. Error analysis from scanned data

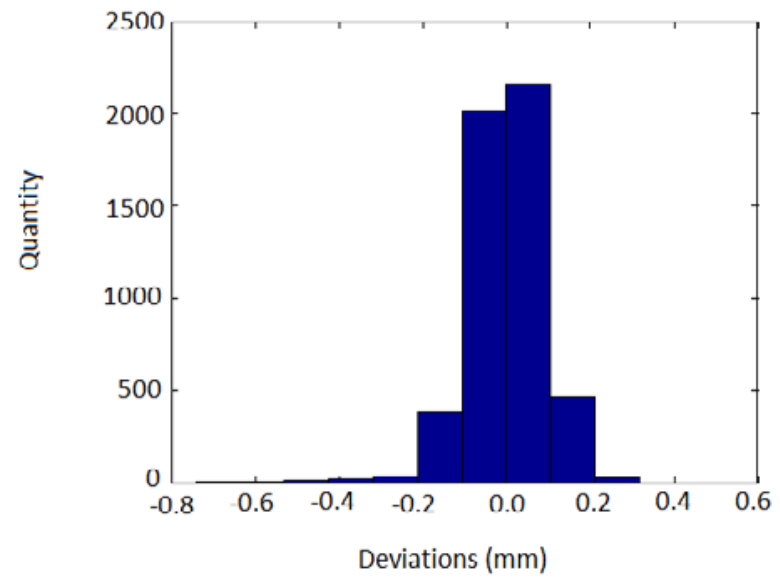

Figure 3. Histogram with Plane A data

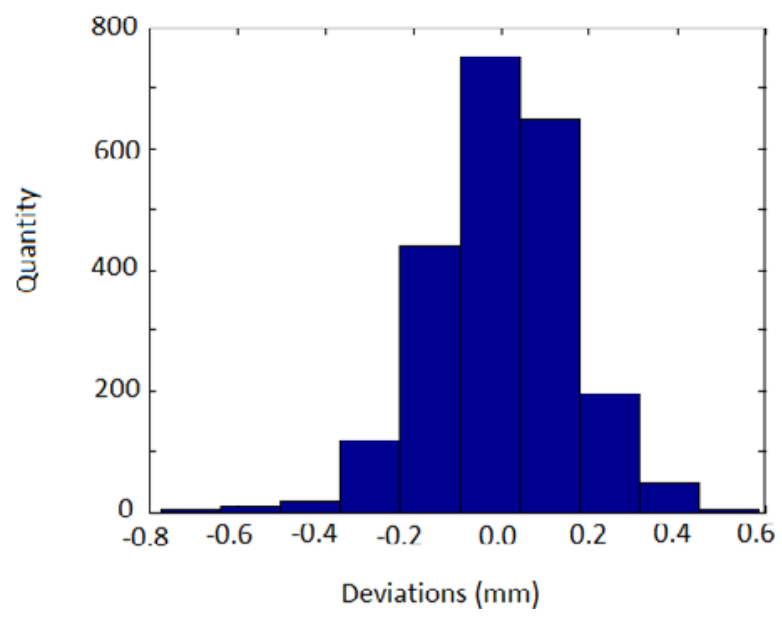

Figure 4. Histogram with Plane B deviations

Verification of normality assumption was performed by building the normal probability plot for planes A and B. These graphs can present also the points that can be considered as outliers and are showed in figures 5 and 6. 


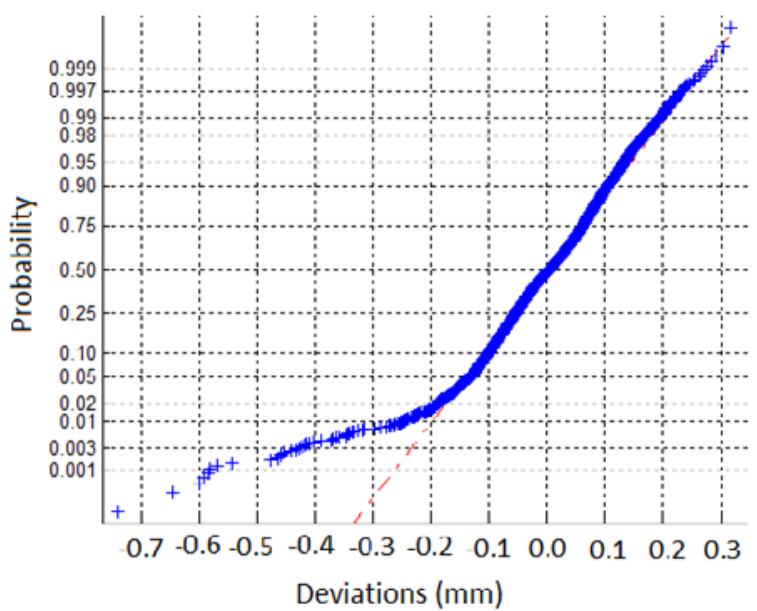

Figure 5. Normal probability plot of Plane A data

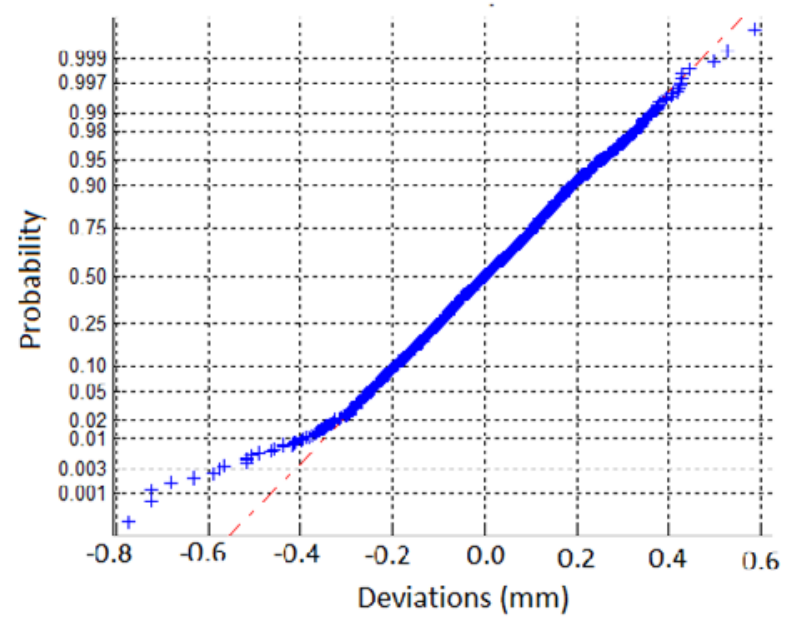

Figure 6. Normal probability plot of Plane B deviations

It was observed that the hypothesis of Normal Probability Distribution (NPD) of deviations in respect to planes A and B can not be accepted, since the data points have a pronounced divergence in respect to the straight line stablishing the NPD. This behaviour is strongly influenced by the negative deviations that appears to be outliers, e.g., resulted from other sources of errors like from laser light reflection.

It was used a statitical filter by building boxplot graphs presented in figures 7 and 8 .

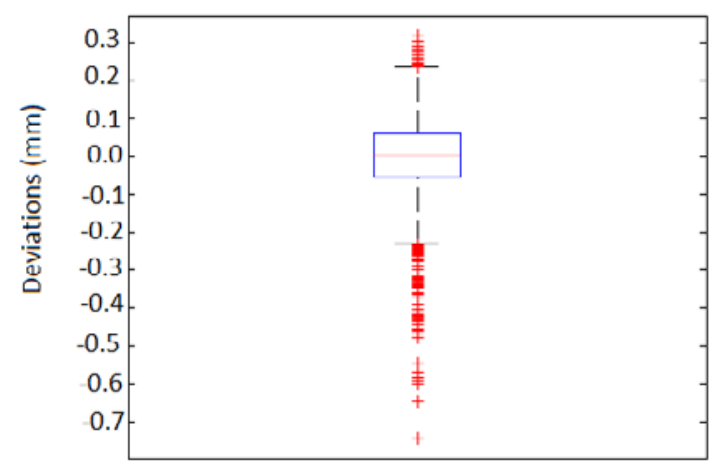

Figure 7 - Boxplot of deviations of plane A showing outliers

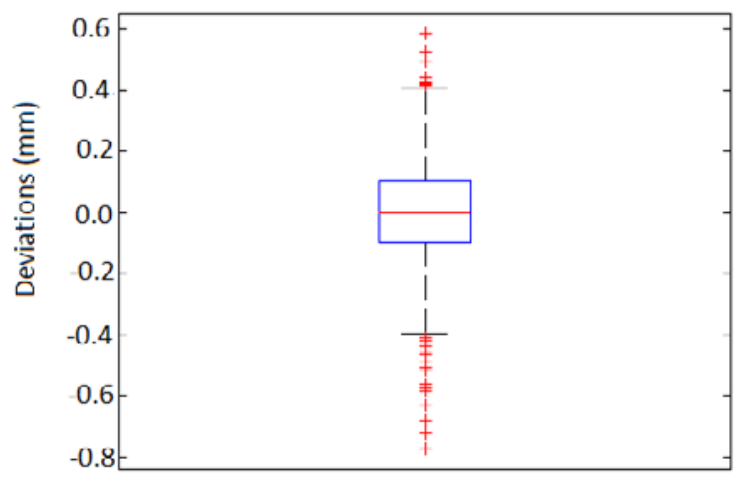

Figure 8 - Boxplot of deviations of plane B showing outliers

As showed, there are some points considered as outliers, marked in red, that must be removed before make any statement of performance. After removing these deviations, Table 1 shows the statistics for deviation in respect to planes $\mathrm{A}$ and $\mathrm{B}$, after removing outliers from results.

Table 1. Statistics from deviations of planes A and B

\begin{tabular}{|c|c|c|}
\hline Plane & A & B \\
\hline Min $(\mathrm{mm})$ & -0.230 & -0.399 \\
\hline Max $(\mathrm{mm})$ & 0.234 & 0.410 \\
\hline Range $(\mathrm{mm})$ & 0.463 & 0.808 \\
\hline Mean $(\mathrm{mm})$ & 0.004 & 0.003 \\
\hline Std deviation $(\mathrm{mm})$ & 0.079 & 0.145 \\
\hline
\end{tabular}

Standard deviation and range for deviations in respect to planes A and B can be considered as performance parameters. Thus, it was found a standard deviation of $0.079 \mathrm{~mm}$ and $0.145 \mathrm{~mm}$, close to the stated by the manufacturer for the same scanning conditions, e.g., $0.127 \mathrm{~mm}$.

For angle measurements, an algorithm was built in MatLab to determine the angle between the planes A and $\mathrm{B}$, fitted by least squares method. It was determined an angle of $90.8261^{\circ}$, resulting in an error of $0.825^{\circ}$ or 49'30". The standard deviation was determined as $0.354^{\circ}$ or $21^{\prime} 14$ '". This error could be affected by the gauge orientation during measurement, together with the uncertainty in plane adjustment.

\section{Conclusions}

A performance verfication of low cost 3D laser scanner was presented involving the measurement of a granite square. Data from scanning of two planes of the square were used to fit planes and to calculate the deviations points-plane and determine the angle.

The square gauge proved suitable to check performance of the low cost scanners, since its has low cost and very small uncertainty in plane surfaces compared to scanner one, $0.211 \mathrm{~mm}$ and $0.02 \mathrm{~mm}$, respectively. A filtering 
method with boxplot graph proved simple and well known technique to eliminate outliers and reestablish Normal probability distribution. The standard deviation was adopted as scanner performance parameter and it was determined as $0.145 \mathrm{~mm}$ at plane $\mathrm{B}$, close to the values established by manuafacturer, $0.127 \mathrm{~mm}$ for the same scanning conditions.

Research efforts may still investigate the application of other filtering methods to supress noise in scanning measurements.

\section{Acknowledgements}

The authors would like to acknowledge the Conselho Nacional de Desenvolvimento Científico e Tecnológico (CNPq), Brazil, and the Fundação de Apoio à Pesquisa do Distrito Federal (FAPDF), Brazil, for financing this work.

\section{References}

[1] Barbero, B.R., Ureta, E.S. Comparative study of different digitization techniques and their accuracy. Computer Aided Design, 43 (2): 188-206, 2011.

[2] Lartigue, C., Contri, A., Bourdet, P. Digitised point quality in relation with point exploitation. Measurement, 32: 193-203, 2002.

[3] Zúniga, L.D.O., Piratelli-Filho, A. Method to check performance of laser scanners using tridimensional artifact. Proc. of COBEM, Ribeirão Preto, Brazil, November 3-7, 2013.

[4] Besic, I., Gestel, N.V., Kruth, J-P., Bleys, P., Hodolic, J. Accuracy improvement of laser line scanning for feature measurements on CMM. Optics and Lasers in Engineering, 49 (2011): 1274-1280.

[5] Gestel, N.V., Cuypers, S., Bleys, P., Kruth, J-P. performance evaluation test for laser line scanners on CMMs. Optics and Lasers in Engineering, 47: 336342, 2009. 